\title{
Robotic grippers for large and soft object manipulation
}

\author{
Kristóf Takács \\ Antal Bejczy Center of Intelligent Robotics \\ Óbuda University \\ Budapest, Hungary \\ kristof.takacs@irob.uni-obuda.hu \\ Lars Bager Christensen \\ Danish Technological Institute (DTI) \\ Taastrup, Denmark \\ lbc@dti.dk
}

\author{
Alex Mason \\ Norwegian University of Life Science (NMBU) \\ Ås, Norway \\ alex.mason@nmbu.no
}

\begin{abstract}
Grasping has always been considered a key domain of cyber-physical systems, through which action physical interaction can be achieved. This paper presents a systematic review of the state-of-the-art robotic soft object gripping solutions aimed for the food-industry, focusing on red meat handling. A categorized analysis about the currently used grippers is provided, that could be used or adapted to robotic meat-processing. The paper enlists various solutions and gripping principles for low-payload applications too, although the emphasis is on the classic shapelocking and force-locking grippers that are potentially capable of grasping and manipulating heavier specimens. The purpose of the scientific literature survey is mainly to identify exceptional and/or remarkable gripper-designs, or completely new gripping concepts, while the patent research presents complete, commercially available solutions.
\end{abstract}

Index Terms - robotic gripper, meat grasping, meat processing, soft-tissue manipulation

\section{INTRODUCTION}

Successful gripping of an object by a cyber-physical system requires an adequate grasping mechatronic structure, some kind of sensory input, and adjacent control (i.e., eye-hand coordination) [1]. This article focuses on the former. Grasping and gripping are typical tasks in both industrial and service robotic applications, where physical interaction is required [2]. Although the two terms are often used as synonyms, grasping initially referred to the action of seizing and holding an object, as if by clasping with the fingers, while gripping referred to grabbing something and holding it firmly. Since one basic purpose of automation is to replace human labor in repetitive or dangerous tasks, the development of safe and effective grippers

This work has received funding from the European Union's Horizon 2020 research and innovation programme under grant agreement No 871631, RoBUTCHER (A Robust, Flexible and Scalable Cognitive Robotics Platform)

Kristof Takacs acknowledges the financial support of Óbuda University Doctoral School of Applied Informatics and Applied Mathematics.

T. Haidegger is supported through the New National Excellence Program of the Ministry of Human Capacities.

T. Haidegger is a Bolyai Fellow of the Hungarian Academy of Sciences, his research is partially supported by the EFOP-3.6.2-16-2017-00016 project. has been an important factor from the beginning. Replacing the human hand in its complexity (dexterity, payload, degrees of freedom, feedbacks etc.) with a robotic gripper is technically very challenging, however in most cases, that is not even required, since robotic grippers can outperform humans in any important individual metrics of a given application (e.g, speed, payload or precision).

Similarly to other aspects of robotic applications, gripping technology is quickly developing. In classical factory automation the gripper-robot usually only had to be able to grasp one kind of rigid target object with a known shape at a predefined place, however modern robotic applications often require much more complicated gripping solutions. New challenges include varying and undefined target-object shapes, fragile, slippery or highly elastic target materials, and only roughly defined or constantly changing environments [3]. Many of these new circumstances require the grippers to coordinate with external devices such as 3D cameras, other robot arms or even human operators (collaborative robotics). These tendencies led to the appearance of the so called intelligent grippers, which may use cloud-based computing [4], may have built-in sensors (for force-feedback, slip-detection etc.) and integrated closed control loop [5], [6], [7].

Within the field of soft tissue gripping, this study focuses on the red meat sector, specifically on the gripping and manipulation of larger masses of swine meat or guts, which is necessary at slaughter house automation. The purpose of this paper is to identify suitable concepts for the gripperdesign process in the RoBUTCHER project, which aims to develop the first entirely automated slaughterhouse cell [8]. The gripping-tasks in that project include the grasping of the limbs, and the grasping of the trachea (for the removal of the internal organs), both cases require a firm grip on a soft, slippery, roughly cylindrical targets.

Considering swines, the typical diameter of the limbs at that point is $4-7 \mathrm{~cm}$, the weight of a cut-off forelimb is $7-10$ 
TABLE I

CHARACTERISTICS OF ROBOTIC GRIPPERS AND THEIR RELEVANCE IN MEAT-GRIPPING APPLICATIONS (3: MOST IMPORTANT, 1: LEAST IMPORTANT)

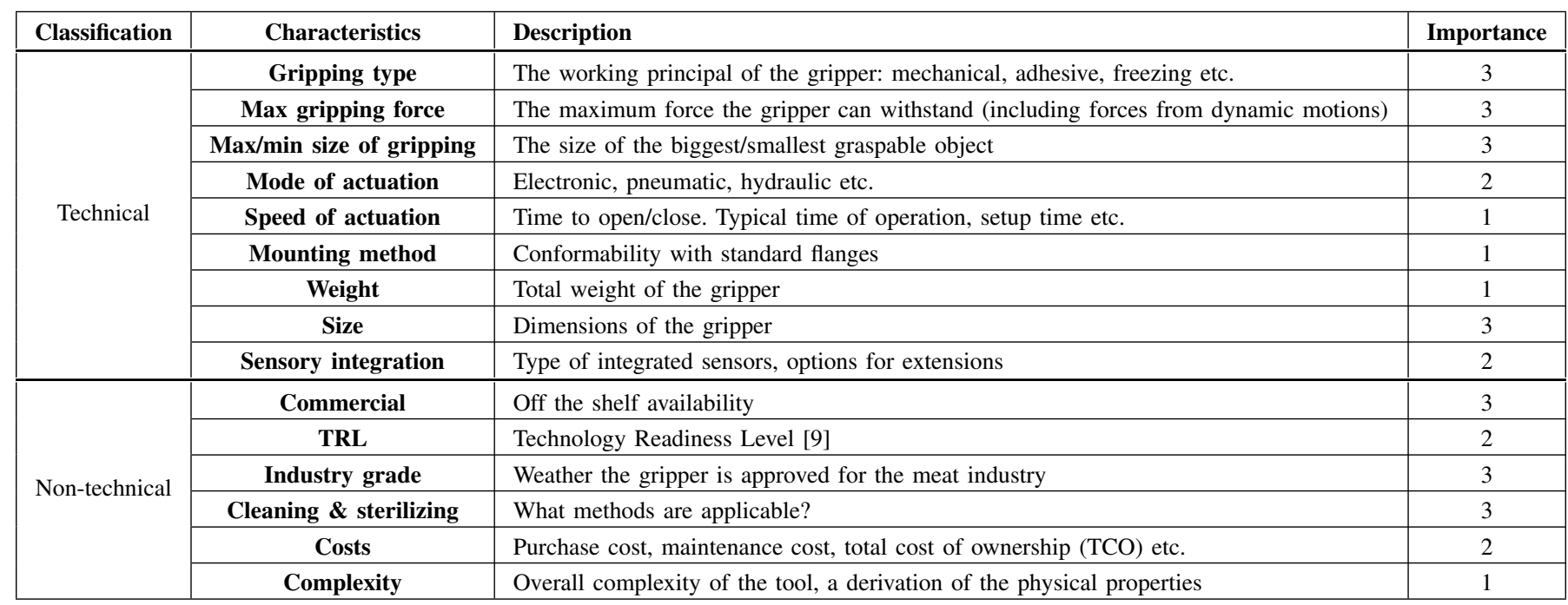

$\mathrm{kg}$, while the ham is usually $8-14 \mathrm{~kg}$. The handling of the entrails (or the evisceration itself) and the remaining carcass may also be automated, thus requiring robotic manipulation. The intestines are usually dropped/pulled into a bowl, while the pluck should be hung on a rack for examination. The practical gripping point in that case is the trachea (that can withstand the ca. $15 \mathrm{~kg}$ weight of all the entrails), which is a cylindrical, slippery and highly elastic gripping target. The remaining half-carcasses usually weigh between $15-20 \mathrm{~kg}$, the most common grippers for their manipulation are suction cups.

The two main challenges regarding robotic meat-gripping applications are hygiene and the natural variance of size, shape and weight of animals. This paper focuses on the gripperdesigns and innovative mechatronic solutions, thus the issue of hygiene and disinfection is out of scope, however the latter was an important aspect during the research.

\section{Methods}

To provide a complete overview of the landscape, the recent published results of soft material grippers were reviewed, based on the PRISMA methodology (Fig 1 shows the PRISMA-flowchart). Using such an approach is very efficient to identify papers in relevant databases, but it must be mentioned that some might be missed even if they are relevant, when they are not published in the selected databases.

Google Scholar, NCBI Pubmed, IEEE Xplore and general search engines were explored for research to collect possible results published in the last 5 years with the following key terms (patents excluded):

- "Meat gripper" OR

- "Soft tissue gripping" OR

- "Meat processing gripper" OR

- "Red meat processing robot" OR

- "Robotic pig slaughter" OR

• "Pig slaughter automation".

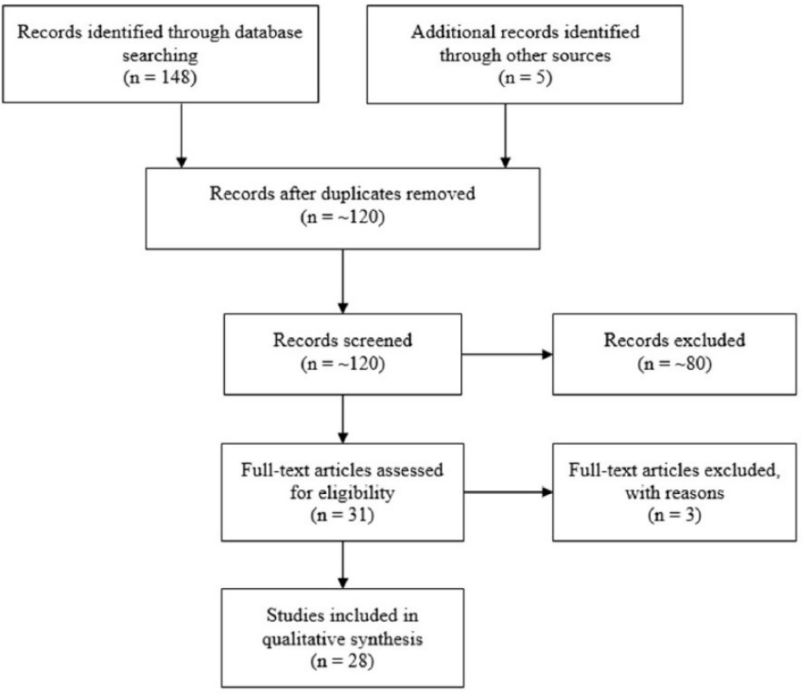

Fig. 1. PRISMA flow-chart about the scientific paper research.

It was revealed during the research that out of the 6 keywords, only the first 3 provided relevant results, the latter 3 failed to reveal new and important papers (also the NCBI database seemed to be irrelevant from the chosen aspect). Publications presenting innovative gripper-designs were the primary focus, however, papers about software and simulation related to robotic grasping were also included eventually in the detailed review process. As a result, there are records that are not intended to present innovative gripper-designs, but found to be potentially useful when choosing or designing whole robotic gripping applications.

The goal was to identify new perspectives and unique ideas about the whole soft tissue (red meat) gripping process. Thus, the collection of scientific papers chosen for thorough review 

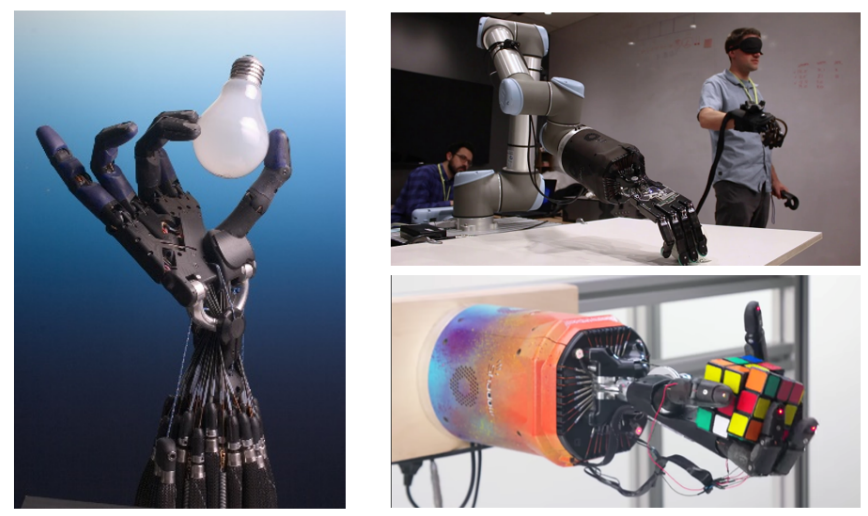

Fig. 2. Advanced robot-hands [10], [11], [12].

includes gripper-designs based on unusual principles, softtissue related software, simulations and surveys as well.

The final set of included research papers was established with the following method:

1) Search for the keywords in the mentioned collections with the following restrictions:

- Published in 2016 or later;

- Patents excluded (to avoid further duplications);

- Arranged based on relevance.

2) Save the relevant papers. Examples of discarded topics that came up frequently:

- Micro-grippers;

- Studies about "biological grippers" (e.g, different types of tongues);

- Grippers for packaged food (typically simple suction-cups);

- Papers only mentioning, but not focusing on grippers.

3) Remove duplicates.

Further, a patent database search was ordered from WIPO (World Intellectual Property Organisation), within the classes: b23 (machine tools for shaping) OR b25 (hand tools for shaping) OR g05 (controlling or regulating) OR b29c (shaping or joining of plastics) OR b65g (transport or storage devices) OR b66c (devices for cranes) OR f15b (systems acting by means of fluids in general) OR f16b (devices for fastening, e.g., Clamps) OR g01 (measuring or testing) OR h01 (basic electric elements) OR h02 (generation, conversion or distribution of power) OR h04 (electric communication technique) with the following relevant search key terms:

- Gripping OR

- Clamping OR

- Damping OR

- Finger OR

- End-of-Arm OR

- Robot OR

- Robotic.

The gross list consisted of more than 200 candidates dating back to 1983. It covers a wide range of application areas, with
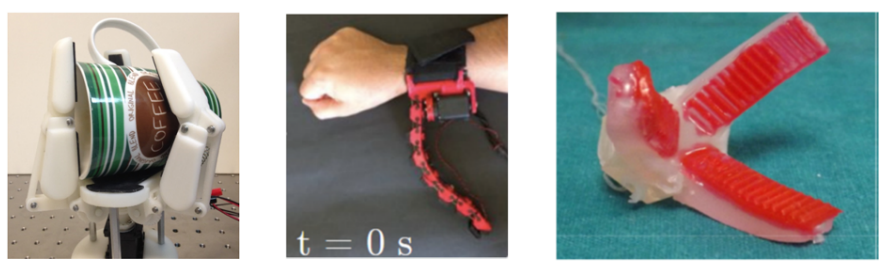

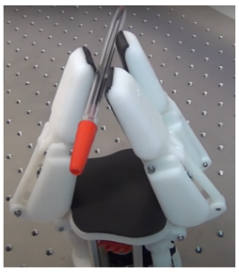

a

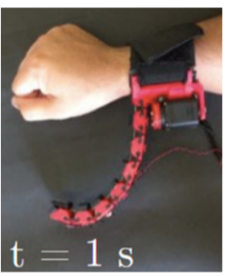

b

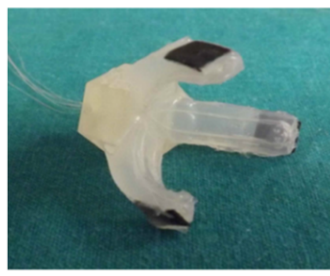

c
Fig. 3. Examples for underactuated, finger-based grippers.

some deemed only to be relevant for industries other than the food industry. Excluding such specific industry applications, 98 remaining applications were assessed for relevance for the red meat sector. The assessment was made from two points of view: commercial availability and relevance in addition to potential impediment of freedom to operate.

\section{ROBOTIC GRIPPERS FOR THE MEAT INDUSTRY}

\section{A. Principles}

Despite the efforts to exclude the irrelevant design-ideas during database searches, some gripping-principles turned out to be unsuitable for our domain due to different reasons still showed up in the final collection. These methods usually adopt principles that can work well on a small workpiece (e.g; in laparoscopic surgery) but cannot be scaled up to the application in question (e.g; grippers using Coanda-effect or capillary-effect) [13], [14], [15], [16].

Before the review of the related designs and papers, the main required parameters of a general robotic gripper were identified. These attributes are classified in Table I. based on their relevance to the food industry and meat-gripping applications. Usually, not all of the important technical metrics are presented numerically in the reviewed papers (nor in the patents), thus it can not always be certainly decided if a specific design is suitable for a given task. As a consequence, this paper focuses primarily on the principles and ideas behind the presented gripper, and only secondly on the constructed gripper itself.

\section{B. Gripper designs}

Probably the most complex type of robotic grippers are the advanced prosthetic hands and hand-like grippers. The basic goal here is to be as similar to a biological hand as possible, thus the purpose of such grippers can be either to replace a human hand, or to be applied onto a robot arm to carry out complex gripping tasks. Although the motivations in the two cases are different, the results—-regarding the finger-shaping or 


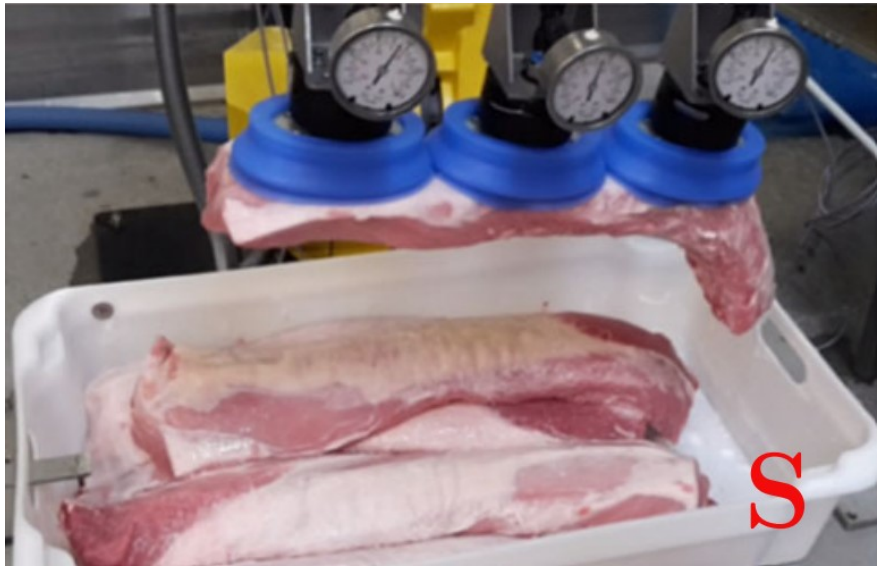

Fig. 4. Example of raw red meat handling with suction cups and pressure control [25].

the actuation-method-are generally similar, thus the designideas behind prosthetic hands can often be used for industrial grippers and vice versa. However, despite all the effort to replicate the human hand, its complexity and efficiency still seems close to impossible to reach [17].

There are several mechanical models of the human hand, which makes the replication even more difficult. The rotational bending joints in the fingers are definite (3 joints on each finger, 15 Degrees of Freedom (DoF) altogether), however the number of actuated and non-actuated DoFs in the carpus and the adduction/abduction DoFs of the finger-joints are less exact. Most models estimate the total DoFs of a human hand is between 25 and 30. The most accepted models are of 25 DoF [18] [19] and 27 DoF [20], [21]. The most advanced hand-like gripper designs may have up to 5 fingers, as many DoFs as a human hand, have implemented tactile-feedback and sometimes even skin-mimicking sensorized coating (Fig 2). Although these systems are usually far too complex and complicated for casual food-industry applications, a strong enough design could probably be useful for complex tasks. The payload of this type of gripper is, however, usually only 1-2 kg; the highest found was $12 \mathrm{~kg}$. The most promising way to adjust such a gripper-type for meat-gripping would be a "reduced hand" with only 2-3 fingers, less DoF, but a similar grasping method [22], [10], [23], [11], [24].

Another type of grippers could be interpreted as a simplified hand, namely the grippers with under-actuated fingers (Fig 3 ). These grippers have significantly more DoFs (sometimes infinite) than actuators, thus the bending-trajectory of the fingers during grasping depends on the shape of the targetobject. This behaviour makes underactuated grippers a popular choice for meat and soft tissue manipulation tasks, since it can eliminate the effect of biological variation by design (e.g, the diversity of the diameter of the legs as gripping-targets). Most of the solutions are based on tendons actuating more than one joints. Furthermore, a publication about a mathematical approach was identified to alternate the behaviour of underactuated grippers by modifying the friction of the joints [10].

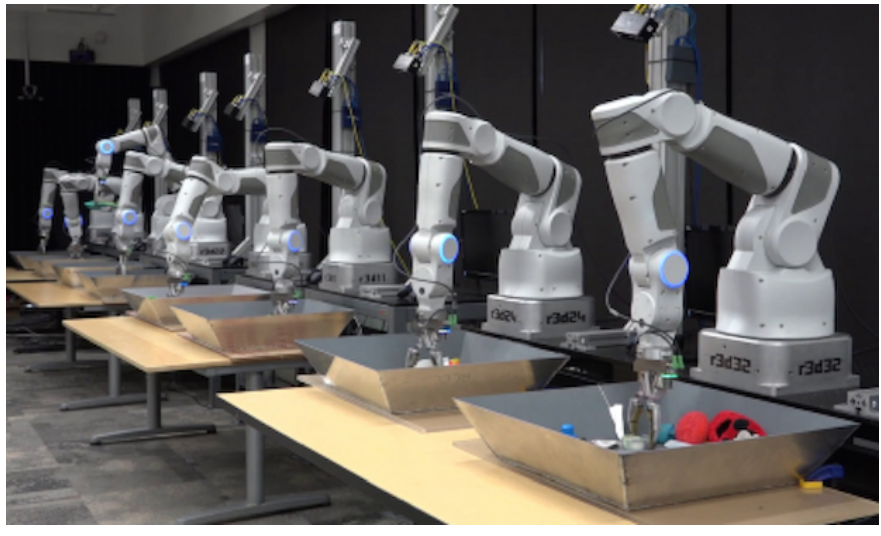

Fig. 5. The Grasp2Vec project of Google, where several identical robots generated data by trying to grasp ordinary objects for months [32]

Low et al. described a tele-manipulated 3D printed gripper with four sensorized fingers and a sensor-enabled haptic-glove [26]. A 3D printed gripper is usually too weak for meatgripping applications (also the food safety of 3D printing materials is an issue); however, the haptic-glove and telemanipulated gripper (or knife) approach could bring advances to butchery (e.g, safety and work-environment improvements) [10], [27] [28]. There are also several patents presenting more complex designs with usually 1-3 under-actuated fingers [29], [30], [31].

One more frequently used approach for soft-tissue manipulation is a design that employs stick-like fingers moving (more or less) radially. The advantage of these grippers is their simplicity: one motor can move all the fingers, forcecontrol is relatively easy to implement, and the cleaning of the fingers is more simple (since there are usually no electronic or mechanical parts inside the fingers). The fingers can be made of many food industry-grade materials, and can have complex shapes; however, these designs are sub-optimal for the grasping of flexible cylindrical objects, they work best with highly deformable target-objects [16], [33].

A completely different approach for the manipulation of huge masses of meat products is the use of suction cups. Usually several suction cups are connected in parallel to increase load capacity and to achieve more secure holding. The advantages of such a gripping system derive from its simplicity: the actuator (vacuum pump) can be placed farther from the robot and its workspace, the number and placement of cups can easily be modified, and the maximum holding force depends mainly only on the power of the pump. However, the utility of vacuum-based grippers are highly limited the circumstances, small differences between target objects can easily prevent the formation suction power and contamination can easily affect the system. Furthermore, a flat and smooth surface is always required under the suction cups, although the grasping of soft and easily deformable targets is easier, since deformation may provide the airtight connection between the target surface and the suction cups (as shown on Fig 4). These designs are usually used for handling big pieces of meat, or 
already packaged meat products [34], [35].

\section{Software and simulations}

The final, yet but still important part of the survey consists of papers presenting software solutions for gripping applications and simulations. These papers usually assume simple grippers, array of suction cups, few DoF fingers or casual 1 DoF grippers made up of only one open-close mechanism (Fig 5).

The usual goal of the presented methods is to find an optimum using virtual mechanical models, artificial intelligence and/or simulation. The subject of the optimization can be either the gripper itself (e.g; finding the optimal length of phalanges or the optimal friction of joints in an under-actuated mechanism) or the gripping movements and trajectories. In most cases with meat gripping applications, the shapes and positions of the target objects and gripping points are known (at least roughly), thus simulation and optimization of the grasping directions and gripping points, or AI-based trajectory planning might be efficient [24], [36], [37], [38], [39], [40], [41], [42].

\section{Discussion}

In this survey several state-of-the-art robotic gripping concepts and gripper designs were examined, while the variations of most popular, but simple grippers that has only two rigid fingers actuated by a simple 1 DoF mechanism were excluded. Robotic meat handling is a complex task due to the biological diversity and the softness of the meat, thus it requires unusual and modern solutions. Advanced robotic hands might be an adequate solution since they might be controlled remotely with implemented force-feedback. They can also be combined with the mentioned AI-based software solutions, however such a system is found to be probably too complex and expensive.

A promising concept for soft tissue manipulation is underactuation. Uderactuated grippers can adapt to the varying shapes and the deforming meat by design, making them a more simple, but still effective solution. Vacuum-grippers with suction cups are suitable for larger masses of meat, however a stable grip requires the use of several smaller suction cups and probably pressure control and monitoring too. Future work on the topic could cover wider range of gripping tasks occurring in the food-industry (different types of meat, grippers for vegetables etc.) and the excluded aspects of meat gripping too, such as cleanability, food-grade materials, control methods etc.

\section{CONCLUSiON}

The intention of this survey was to provide an overview of applicable gripping principles and complete gripper-designs for the RoBUTCHER project. This project aims to produce the first entirely automated pig-slaughtering solution using robotarms and end-effectors. For this, an advanced "intelligent" robotic gripper is required for grasping and manipulating the limbs and organs, and furthermore the carcass has to be secured by a vacuum-gripper solution. This review identified several promising engineering designs and principles for grasping such soft, slippery, but still heavy objects, besides gripping-related software for tactile-feedback, AI-based gripping, and simulations.

\section{REFERENCES}

[1] A. Kovari, J. Katona, and C. Costescu, "Quantitative analysis of relationship between visual attention and eye-hand coordination," Acta Polytech. Hung, vol. 17, pp. 77-95, 2020.

[2] G. Haidegger and I. Paniti, "Episodes of robotics and manufacturing automation achievements from the past decades and vision for the next decade," ACTA POLYTECHNICA HUNGARICA: JOURNAL OF APPLIED SCIENCES, vol. 16, no. 10, pp. 119-136, 2019.

[3] T. Haidegger, P. Galambos, and I. J. Rudas, "Robotics 4.0 - are we there yet?" in 2019 IEEE 23rd International Conference on Intelligent Engineering Systems (INES), 2019, pp. $000117-000124$.

[4] B. Kehoe, D. Berenson, and K. Goldberg, "Toward cloud-based grasping with uncertainty in shape: Estimating lower bounds on achieving force closure with zero-slip push grasps," in IEEE International Conference on Robotics and Automation, 2012, pp. 576-583.

[5] V. Tincani, G. Grioli, M. G. Catalano, M. Garabini, S. Grechi, G. Fantoni, and A. Bicchi, "Implementation and control of the velvet fingers: A dexterous gripper with active surfaces," in IEEE International Conference on Robotics and Automation, 2013, pp. 2744-2750.

[6] J. Li, S. Dong, and E. Adelson, "Slip detection with combined tactile and visual information," in IEEE International Conference on Robotics and Automation (ICRA), 2018, pp. 7772-7777.

[7] D. Dornfeld and C. Handy, "Slip detection using acoustic emission signal analysis," in IEEE International Conference on Robotics and Automation, vol. 4, 1987, pp. 1868-1875.

[8] O. Alvseike, H. Sverdvik, M. O'Farrell, and P. Berg, "Meat factory cell-a concept for the future?" in 63rd International Congress of Meat Science and Technology: Nurturing locally, growing globally. Wageningen Academic Publishers, 2017.

[9] E. H. Conrow, "Estimating technology readiness level coefficients," Journal of Spacecraft and Rockets, vol. 48, no. 1, pp. 146-152, 2011.

[10] V. Kumar, Z. Xu, and E. Todorov, "Fast, strong and compliant pneumatic actuation for dexterous tendon-driven hands," in 2013 IEEE International Conference on Robotics and Automation, May 2013, pp. 15121519.

[11] S. Casley, T. Choopojcharoen, A. Jardim, and D. Ozgoren, "The iris platform - a prostetic device including force sensing," 2014.

[12] I. Akkaya, M. Andrychowicz, M. Chociej, M. Litwin, B. McGrew, A. Petron, A. Paino, M. Plappert, G. Powell, R. Ribas et al., "Solving rubik's cube with a robot hand," arXiv preprint arXiv:1910.07113, 2019.

[13] E. Natarajan, L. W. Hong, M. Ramasamy, C. C. Hou, and R. Sengottuvelu, "Design and development of a robot gripper for food industries using coanda effect," in IEEE 4th International Symposium in Robotics and Manufacturing Automation (ROMA), Dec 2018, pp. 1-5.

[14] J. van den Berg, "Capillary-based gripping for laparoscopic bowel surgery," 2018.

[15] G. Rateni, M. Cianchetti, G. Ciuti, A. Menciassi, and C. Laschi, "Design and development of a soft robotic gripper for manipulation in minimally invasive surgery: A proof of concept," Meccanica, vol. 50, no. 11, pp. 2855-2863, Nov. 2015.

[16] J.-H. Low and C.-H. Yeow, "Rod-based Fabrication of Customizable Soft Robotic Pneumatic Gripper Devices for Delicate Tissue Manipulation," JoVE Scientific peer-reviewed video journal, no. 114, Aug. 2016.

[17] G. Salvietti, "Replicating human hand synergies onto robotic hands: A review on software and hardware strategies," Frontiers in neurorobotics, vol. 12, p. 27, 2018.

[18] E. P. Pitarch, J. Yang, and K. Abdel-Malek, "Santos"TM hand: A 25 degree-of-freedom model," in Digital Human Modeling for Design and Engineering Symposium. SAE International, jun 2005. [Online]. Available: https://doi.org/10.4271/2005-01-2727

[19] A. Savescu, L. Cheze, X. Wang, G. Beurier, and J.-P. Verriest, "A 25 degrees of freedom hand geometrical model for better hand attitude simulation," SAE Technical Papers, vol. 113, 2004.

[20] G. ElKoura and K. Singh, "Handrix: Animating the human hand," Proceedings of the 2003 ACM SIGGRAPH/Eurographics Symposium on Computer Animation, pp. 110-119, 2003.

[21] G. Dewaele, F. Devernay, and R. Horaud, "Hand motion from 3d point trajectories and a smooth surface model," in Computer Vision - ECCV 2004. Springer Berlin Heidelberg, 2004, pp. 495-507. 
[22] J. Spiliotopoulos, G. Michalos, and S. Makris, "A reconfigurable gripper for dexterous manipulation in flexible assembly," Inventions, vol. 3, no. 1, 2018. [Online]. Available: https://www.mdpi.com/2411$5134 / 3 / 1 / 4$

[23] H. Chinbe, T. Yoneyama, T. Watanabe, K. Miyashita, and M. Nakada, "Finger-attachment device for the feedback of gripping and pulling force in a manipulating system for brain tumor resection," International journal of computer assisted radiology and surgery, vol. 13, no. 1, pp. 3-12, 2018.

[24] E. Misimi, A. Olofsson, A. Eilertsen, E. Oye, and J. Mathiassen, "Robotic handling of compliant food objects by robust learning from demonstration," in IEEE/RSJ International Conference on Intelligent Robots and Systems (IROS), 2018, pp. 6972-6979.

[25] T. B. Jørgensen, S. H. N. Jensen, H. Aanæs, N. W. Hansen, and N. Krüger, "An Adaptive Robotic System for Doing Pick and Place Operations with Deformable Objects," Journal of Intelligent \& Robotic Systems, vol. 94, no. 1, pp. 81-100, 2019.

[26] J. H. Low, W. W. Lee, P. M. Khin, N. V. Thakor, S. L. Kukreja, H. L. Ren, and C. H. Yeow, "Hybrid tele-manipulation system using a sensorized 3-d-printed soft robotic gripper and a soft fabric-based haptic glove," IEEE Robotics and Automation Letters, vol. 2, no. 2, pp. 880 887, 2017.

[27] G. Salvietti, I. Hussain, M. Malvezzi, and D. Prattichizzo, "Design of the passive joints of underactuated modular soft hands for fingertip trajectory tracking," IEEE Robotics and Automation Letters, vol. 2, no. 4, pp. 2008-2015, 2017.

[28] D. A. Tomzik and X. W. Xu, "Requirements for a cloud-based control system interacting with soft bodies," in 24th International Conference on Mechatronics and Machine Vision in Practice (M2VIP), Nov 2017, pp. $1-5$.

[29] T. Moyer, E. L. Faulring, and J. J. Santos-Munne, "One motor finger mechanism," Jun. 25 2013, uS Patent 8,470,051.

[30] M. J. DeLouis, "Robotic hand with conformal finger," Mar. 31 2015, uS Patent 8,991,884.

[31] J. Curhan, T. Womersley, and J. A. Lessing, "Robotic gripper for handling meat products," 2019, uS Patent App. 16/053,213.

[32] E. Jang, C. Devin, V. Vanhoucke, and S. Levine, "Grasp2vec: Learning object representations from self-supervised grasping," arXiv preprint arXiv:1811.06964, 2018.

[33] G. Endo and N. Otomo, "Development of a food handling gripper considering an appetizing presentation," in IEEE International Conference on Robotics and Automation (ICRA), 2016, pp. 4901-4906.

[34] T. B. Jørgensen, B. R. Hansen, M. M. Pedersen, N. Krüger, and N. W. Hansen, "A flexible suction based grasp tool and associated grasp strategies for handling meat," in 4th International Conference on Mechatronics and Robotics Engineering, 2018, p. 43-47.

[35] J. A. Hilario Poma, J. Galvan, I. Quispe, R. Manzanares Grados, and F. Cordova, "Design of a 2-dof delta robot for packaging and quality control of processed meat products," in Latin American Robotic Symposium, Brazilian Symposium on Robotics (SBR) and Workshop on Robotics in Education (WRE), 2018, pp. 201-206.

[36] B. J. A. Tony and M. S. Alphin, "Finite element analysis to assess the biomechanical behavior of a finger model gripping handles with different diameters," Biomedical Human Kinetics, vol. 11, no. 1, pp. 69-79, 2019.

[37] H. Liang, X. Ma, S. Li, M. Görner, S. Tang, B. Fang, F. Sun, and J. Zhang, "Pointnetgpd: Detecting grasp configurations from point sets," in International Conference on Robotics and Automation (ICRA), 2019, pp. 3629-3635.

[38] T. B. Jørgensen, S. H. N. Jensen, H. Aanæs, N. W. Hansen, and N. Krüger, "An Adaptive Robotic System for Doing Pick and Place Operations with Deformable Objects," Journal of Intelligent \& Robotic Systems, vol. 94, no. 1, pp. 81-100, Apr. 2019.

[39] S. Iqbal, J. Tremblay, T. To, J. Cheng, E. Leitch, A. Campbell, K. Leung, D. McKay, and S. Birchfield, "Toward sim-to-real directional semantic grasping," 2019.

[40] M. Adjigble, N. Marturi, V. Ortenzi, V. Rajasekaran, P. Corke, and R. Stolkin, "Model-free and learning-free grasping by local contact moment matching," in IEEE/RSJ International Conference on Intelligent Robots and Systems (IROS), 2018, pp. 2933-2940.

[41] C. Eppner, A. Mousavian, and D. Fox, "A billion ways to grasp: An evaluation of grasp sampling schemes on a dense, physics-based grasp data set," 2019.

[42] T. Bo Jørgensen, K. Debrabant, and N. Krüger, "Robust optimization of robotic pick and place operations for deformable objects through simulation," in 2016 IEEE International Conference on Robotics and Automation (ICRA), 2016, pp. 3863-3870. 